\title{
The Effect of Stopping Smoking on Disease Activity in Rheumatoid Arthritis (RA). Data from BARFOT, a Multicenter Study of Early RA
}

\author{
Maria L.E. Andersson ${ }^{*}$, , Stefan Bergman ${ }^{1,2}$ and Maria K. Söderlin ${ }^{1}$ for the BARFOT Study Group ${ }^{\S}$ \\ ${ }^{I} R \& D$ Center, Spenshult Rheumatology Hospital, 31392 Oskarström, Sweden \\ ${ }^{2}$ Department of Rheumatology, IKVL, Lund University, Lund, Sweden
}

\begin{abstract}
Objective: We studied the effect of stopping smoking on disease activity in patients with RA.
Methods: Between 1992 and 2005, 2,800 adult patients were included in the BARFOT early RA study in Sweden. Disease Activity Score 28 joints (DAS28), C-reactive protein (CRP), Health Assessment Questionnaire (HAQ), rheumatoid factor (RF), anti-CCP, general health and pain visual analog scales (VAS), EULAR response and treatment were registered at inclusion and at follow-up 2, 5 and 8 years. In 2010, a self-completion postal questionnaire was sent to 2,102 patients, enquiring about lifestyle factors, including cessation of smoking.

Results: A total of 1,460 adult RA patients with disease duration $\leq 2$ years were included in this study. Seventeen percent smoked in 2010. In total, 127 patients stopped smoking after inclusion in the study. Smoking cessation after inclusion in the study was negatively associated with EULAR good outcome at 8 years (OR 0.44, 95\% CI $0.22-0.86, p=0.02$ ), controlled for age, disease duration, sex, socioeconomic class, smoking status, RF, and DAS28 at inclusion.

Conclusion: Seventeen percent of the RA patients smoked in 2010 in this large Swedish RA cohort. Stopping smoking after onset of RA did not change the poor prognosis of smokers with RA, but all RA patients need to stop smoking because of the high risk of cardiovascular mortality and morbidity and the association of smoking with vasculitis and noduli in RA.
\end{abstract}

Keywords: Smoking, cessation of smoking, stopping smoking, rheumatoid arthritis, epidemiology.

\section{INTRODUCTION}

Rheumatoid arthritis (RA) is a chronic inflammatory disease of the joints leading, if untreated, to permanent joint destruction. Smoking has been identified as the major environmental agent causing RA, interacting with anti-CCP antibodies, shared epitope and the gene PTPN22 [1]. Smoking has also been shown to be a risk factor for poor response in RA patients treated with disease-modifying antirheumatic drugs (DMARDs) [2-7] and with anti-tumor necrosis factor (anti-TNF) treatment [8-12].

A recently published study from the CORRONA registry in the US did not show any effect of stopping smoking after inclusion in the registry [13]. A study from the German Early Arthritis Cohort showed that RA patients who stopped smoking after onset of disease had highest DAS28 (Disease Activity Score 28, joints; www.das-score.nl), highest baseline CRP (C-reactive protein) levels, highest ESR

*Address correspondence to this author at the R\&D Center, Spenshult Rheumatology Hospital, 31392 Oskarström, Sweden;

Tel: +46-35-2635000; Fax: +46-35-2635255;

E-mail: maria.andersson@spenshult.se

${ }^{\S}$ Members of the BARFOT study group: Sofia Ajeganova, Maria Andersson, Valentina Bala, Stefan Bergman, Kristina Forslind, Ingiäld Hafström, Catharina Keller, Ido Leden, Bengt Lindell, Ingemar Petersson, Christoffer Schaufelberger, Björn Svensson, Maria Söderlin, Annika Teleman, Jan Theander, and Anneli Östenson. (erythrocyte sedimentation rate) values, most severe pain, and the highest number of comorbid conditions as compared to current smokers, previous smokers, and those who had never smoked [14]. We have previously reported that $20 \%$ of patients with early RA smoked in 2004, in this same cohort [2], but the current prevalence of smoking in Swedish RA patients is not known.

Our aim was to determine whether stopping smoking after onset of RA had any effect on disease activity in a large multicenter longitudinal observational study of RA patients in southern Sweden (the BARFOT study). Our hypothesis was that the patients who stopped smoking after onset of RA would have lower disease activity and better function than patients who continued smoking. We also wanted to determine whether the time point of stopping smoking before inclusion in the study had any effect on disease activity at inclusion.

\section{METHODS}

During the period 1992-2005, 2,800 patients $>18$ years of age were enrolled in the BARFOT (Better AntiRheumatic FarmacOTherapy) study, a multicenter longitudinal observational study of patients with early RA in southern Sweden [2, 15-17]. All the patients fulfilled the American College of Rheumatology RA classification criteria from 1987 [18].

Disease activity was evaluated at inclusion (i.e. at the enrolment in the study) and at 2, 5 and 8 years. The number 
of swollen joints (28-joint count) (SJC), number of tender joints (28-joint count) (TJC), CRP, ESR, the Swedish version of the Stanford Health Assessment Questionnaire (HAQ) $[19,20]$, and visual analog scale (VAS) for pain and general health were measured on every follow-up occasion. The Disease Activity Score using 28-joint count (DAS28) was calculated at inclusion and on every follow-up occasion (www.das-score.nl). Anti-rheumatic treatment in this study was recorded as DMARDs (disease-modifying antirheumatic drugs, that is non-biologics), biologics and glucocorticoids and was registered at each follow-up point. The disease duration was calculated from the start of the symptoms. In this study, patients had to have disease duration of $\leq 2$ years at inclusion to be enrolled in the study.

The DAS28 is a composite score consisting of the number of swollen joints (of 28), tender joints (of 28), ESR, and the patient's global assessment [21]. EULAR (European League Against Rheumatism) response was calculated from the DAS28 scores [21]. The patients were classified into one of three EULAR response groups: no response, moderate response, or good response. A EULAR good responder had to demonstrate an improvement of at least 1.2 units and achieve an absolute score of $<3.2$. A non-responder should show an improvement of $<0.6$, or $>0.6$ and $\leq 1.2$, and have a final DAS28 of $>5.1$ (www.das-score.nl). EULAR response was analysed at 1,2, 5 and 8 years of follow-up. The choice of DMARD treatment in the BARFOT study was left to the discretion of the rheumatologist. Serum antibodies to cyclic citrullinated peptide were analyzed using the Immunoscan-RA ELISA CCP2 test (Euro-Diagnostica, Malmö, Sweden). A titer above 25 units/ml was regarded as positive.

\section{Self-Completion Postal Questionnaire in 2010}

Between March and September 2010, all patients who were still alive in the BARFOT study $(\mathrm{n}=2102)$ received a self-completion postal questionnaire assessing smoking habits, smoking cessation, pack-year data, snuff use, secondhand smoke exposure, alcohol use, diet, pain, medication, comorbidities, height, weight, waist circumference, ADL function, and physical activity. Demographics such as occupation and immigrant status were also recorded. The occupations were coded according to the latest version of the socioeconomic status (Socioekonomisk indelning, SEI) in Sweden. The socioeconomic status was assessed as manual worker, lower white-collar worker, upper white-collar worker, self-employed, and other according to the SEI.

The 2010 self-completion postal questionnaire enabled us to assess smoking retrospectively and also in a crosssectional manner in 2010, enabling us to calculate the current prevalence of smoking in that year in the BARFOT study. The questions "Are you a smoker?" "What year did you start smoking?" "What year did you stop smoking?" "How many cigarettes do you/did you smoke per day?" permitted the calculation of pack-years (one pack-year is consumption of 20 cigarettes a day for one year). All the patients received written information about the questionnaire in 2010, and the ethical committee at Lund University approved the BARFOT study and the postal questionnaire in 2010.

Our primary objective in the study was to study EULAR response at inclusion and at 2, 5 and 8 years of follow-up, and our secondary objectives were to study the absolute DAS28 values, the individual components of the DAS28, and the HAQ stratified according to smoking class at the same follow-up points.

\section{Statistics}

The Mann-Whitney U-test and chi-square test were used as appropriate. The Kruskall-Wallis test was used to assess demographics and disease activity between the patients who had never been smokers, those who were current smokers, and those who had stopped smoking before and after inclusion in the study. Post hoc analyses using standardized residuals were used to detect differences in EULAR response, stratified for the different smoking categories. Spearman's correlation coefficient was used to assess correlation between year of stopping smoking before inclusion and disease activity at inclusion. For the EULAR response at 2,5 and 8 years, only the patients who had stopped smoking before these time points in the "stopped smoking after inclusion" category were analysed, respectively.

A multiple logistic regression analysis was used to assess whether smoking cessation after inclusion in the study was an independent factor for EULAR response. The outcome of the regression analysis was good $v s$ moderate or no EULAR response at 8 years of follow-up. The following variables were entered into the multiple logistic regression model at inclusion: age, disease duration (months), sex, socioeconomic class (manual worker, lower or upper whitecollar worker, self-employed, other), smoking class (never smoker, current smoker, stopped smoking before or after inclusion), RF, and DAS28 at inclusion. In this analysis, patients who had stopped smoking $>7$ years after inclusion were excluded $(n=42)$. The variables entered in the regression analysis were checked for colinearity.

\section{RESULTS}

A total of 1,524/2,102 (73\%) patients answered the selfcompletion postal questionnaire in 2010. Of these, 1,460 patients were $>18$ years of age and had disease duration of $\leq$ 2 years, and these patients were included in this study. The demographic and disease activity data at inclusion in the study for the 1,525 patients who answered the selfcompletion postal questionnaire in 2010 and the 579 patients who did not answer are shown in Table 1. In summary, patients who did not answer the questionnaire had higher DAS28, higher VAS global, and higher SJC at inclusion and they were more often smokers but less often RF positive than the patients who had answered the 2010 questionnaire.

\section{Attrition}

At 3 months, 1,381 patients had complete EULAR response data, and the figures were $1,285,1,342,1,319$, 1,295 , and 795 for follow-up points 6 and 12 months and 1 , 2,5 , and 8 years, respectively. A total of 160 patients $(11 \%)$ had EULAR response data at 15 years. Altogether, $659 / 1,460$ patients (45\%) had complete data on EULAR response at each follow-up point up to 8 years of follow-up. The 659 patients who had complete data did not differ significantly from the 801 patients $(55 \%)$ who did not have complete EULAR response follow-up data at baseline regarding HAQ $(\mathrm{p}=0.59)$, VAS pain $(\mathrm{p}=0.52)$, VAS global 
Table 1. Disease Activity Variables and Demographics at Inclusion in the Study for the Patients who Answered and Did Not Answer the 2010 Postal Questionnaire. Values are Median (Interquartile Range) Unless Otherwise Stated

\begin{tabular}{|c|c|c|c|}
\hline & $\begin{array}{l}\text { Answered the } 2010 \text { Postal Questionnaire, } \\
\text { Baseline at Inclusion in the Study }\end{array}$ & $\begin{array}{l}\text { Did Not Answer the } 2010 \text { Postal Questionnaire, } \\
\text { Baseline at Inclusion in the Study }\end{array}$ & P Value \\
\hline Variable & $\mathrm{N}=1525$ & $\mathrm{~N}=579$ & \\
\hline Age, years & $56(47-65)$ & $58(42-70)$ & 0.06 \\
\hline Female, \% & 70 & 68 & 0.32 \\
\hline HAQ & $0.9(0.5-1.4)$ & $1.0(0.5-1.5)$ & 0.25 \\
\hline VAS global, mm & $46(24-63)$ & $49(27-67)$ & 0.008 \\
\hline VAS pain, $\mathrm{mm}$ & $46(28-65)$ & $49(29-68)$ & 0.09 \\
\hline Number of swollen joints (of 28) & $10(6-14)$ & $10(6-15)$ & 0.03 \\
\hline Number of tender joints (of 28) & $7(3-12)$ & $8(4-13)$ & 0.06 \\
\hline Number of ongoing DMARDs, $\%$ & $80 \%$ & $80 \%$ & 0.37 \\
\hline Ongoing glucocorticoids, $\%$ & 36 & 39 & 0.14 \\
\hline Smoker, $\%$ & 24 & 30 & 0.01 \\
\hline RF, \% & 63 & 58 & 0.02 \\
\hline
\end{tabular}

$\mathrm{HAQ}=$ Health Assessment Questionnaire, VAS $=$ visual analog scale, DMARDs $=$ disease-modifying anti-rheumatic drugs, DAS28 = Disease Activity Score $(28$ joints), ESR $=$ erythrocyte sedimentation rate, $\mathrm{CRP}=\mathrm{C}$-reactive protein, $\mathrm{RF}=$ rheumatoid factor.

$(\mathrm{p}=0.29), \operatorname{CRP}(\mathrm{p}=0.37), \operatorname{ESR}(\mathrm{p}=0.17)$, and $\operatorname{SJC}(\mathrm{p}=$ $0.97)$, but the patients who had complete data had significantly lower DAS28 at baseline (median 5.2 for complete data vs 5.3 for incomplete data $(\mathrm{p}=0.005))$ and had a lower median number of TJC (7 for complete vs 8 for incomplete $(\mathrm{p}=0.01))$.

Fig. (1) shows the flow chart of the study.

\begin{tabular}{|c|c|c|}
\hline Inclusion & $\begin{array}{l}\text { Never smokers } \\
\text { Previous smokers } \\
\text { Current smokers }\end{array}$ & $\begin{array}{l}n=514 \\
n=490 \\
n=358\end{array}$ \\
\hline & & $\downarrow$ \\
\hline \multirow[t]{2}{*}{2 year follow up } & $\begin{array}{l}\text { Never smokers } \\
\text { Previous smokers } \\
\text { Current smokers } \\
\text { Smoke stop }\end{array}$ & $\begin{array}{l}n=514 \\
n=490 \text { (at inclusion) } \\
n=343 \\
n=15 \quad(<2 \text { years after inclusion) }\end{array}$ \\
\hline & & $\downarrow$ \\
\hline 5 year follow up & $\begin{array}{l}\text { Never smokers } \\
\text { Previous smokers } \\
\text { Current smokers } \\
\text { Smoke stop }\end{array}$ & $\begin{array}{l}n=514 \\
n=490 \text { (at inclusion) } \\
n=304 \\
n=54 \quad(<5 \text { years after inclusion) }\end{array}$ \\
\hline 8 year follow up & $\begin{array}{l}\text { Never smokers } \\
\text { Previous smokers } \\
\text { Current smokers } \\
\text { Smoke stop }\end{array}$ & $\begin{array}{l}n=514 \\
n=490 \text { (at inclusion) } \\
n=274 \\
n=84 \quad(<8 \text { years after inclusion) }\end{array}$ \\
\hline
\end{tabular}

Fig. (1). The flow chart of the study. 
At baseline, $31 \%$ of the patients with incomplete data received glucocorticoids as compared to $43 \%$ of the patients with complete data $(\mathrm{p}=0.0001)$, and these differences persisted for up to 2 years of follow-up (data not shown). The patients with incomplete data had more often received DMARDs at baseline and had more often received combination treatment and biologics during the follow-up. These differences persisted for up to 5 years (data not shown).

A total of $514 / 1362(38 \%)$ of the patients had never smoked, 490/1362 (36\%) had smoked previously, and $231 / 1362(17 \%)$ were current smokers in the questionnaire in 2010. 98 patients had missing data on smoking in 2010. A total of 127 patients stopped smoking either during the year of inclusion or after inclusion in the BARFOT study. Three patients stopped smoking after 15 years of follow-up. There were no differences in baseline disease activity variables between the patients who stopped smoking before and after inclusion in the study, current smokers, and those who had never smoked (HAQ, $\mathrm{p}=0.64$; DAS28, $\mathrm{p}=0.69$; VAS pain, $\mathrm{p}=0.26$; VAS global, $\mathrm{p}=0.78$; CRP, $\mathrm{p}=0.07$; ESR, $\mathrm{p}=$ 0.35; SJC, $p=0.06$; TJC, $p=0.29$ ).

\section{Treatment with DMARDs and Biologics}

Anti-rheumatic treatment (ie. DMARDs (non-biologics), biologics and glucocorticoids) was recorded at the follow-up visits. The patients were mainly treated with DMARDs, that is to say non-biologics. The proportion of patients with no DMARD treatment ranged from $21 \%$ at inclusion to $34 \%$ at 15 years of follow-up. The proportion of patients with DMARD monotherapy diminished from $77 \%$ at inclusion to $36 \%$ at 15 years, and the proportion of patients with combination treatment varied from $1.6 \%$ at inclusion to $12 \%$ at 15 years. The proportion of patients treated with biologics increased from $0.4 \%$ at inclusion to $23 \%$ at 15 years. The proportion of glucorticoid treatment varied from $23 \%$ to $39 \%$.

There were no differences in DMARD treatment or glucocorticoid treatment between the different smoking categories for up to 15 years of follow-up, with 3 exceptions. One exception was glucocorticoid treatment at 5 years, where $19 \%$ of those who had never smoked did not receive glucocorticoids as compared to $22 \%$ of current smokers, $25 \%$ of patients who had stopped smoking before inclusion, and $29 \%$ who stopped smoking after inclusion $(\mathrm{p}=0.04)$. Another exception was DMARD treatment at 8 years, where $32 \%$ of never-smokers did not receive DMARDs as compared to $19 \%$ of smokers, $19 \%$ of the patients who stopped smoking before inclusion, and $27 \%$ of the patients who stopped smoking after inclusion $(p=0.04)$. The third exception was glucocorticoid treatment at 8 years; $18 \%$ of never-smokers did not receive glucocorticoids, as opposed to $24 \%$ of current smokers, $27 \%$ of the patients who had stopped smoking before inclusion, and $32 \%$ of the patients who stopped smoking after inclusion $(\mathrm{p}=0.01)$.

\section{The Effect of Cessation of Smoking After Inclusion on Disease Activity}

We could see no clear differences in disease activity between never-smokers, current smokers, and patients who had stopped smoking before or after inclusion-in HAQ, in
VAS pain, in DAS28 (Fig. 2), or in any of its individual variables for up to 8 years of follow-up. Fig. (2). Looking separately at the individual components of the DAS28, the absolute DAS28 values and HAQ at 2, 5 and 8 years for patients who had stopped smoking before these follow-up points, respectively, as compared to the other smoking classes, there was only a significant difference in CRP at 2 years of follow-up, where non-smokers had a CRP of 10, smokers 11, patients having stopped smoking before inclusion 11 and patients having stopped smoking $<2$ years after inclusion $12(\mathrm{p}=0.03)$.

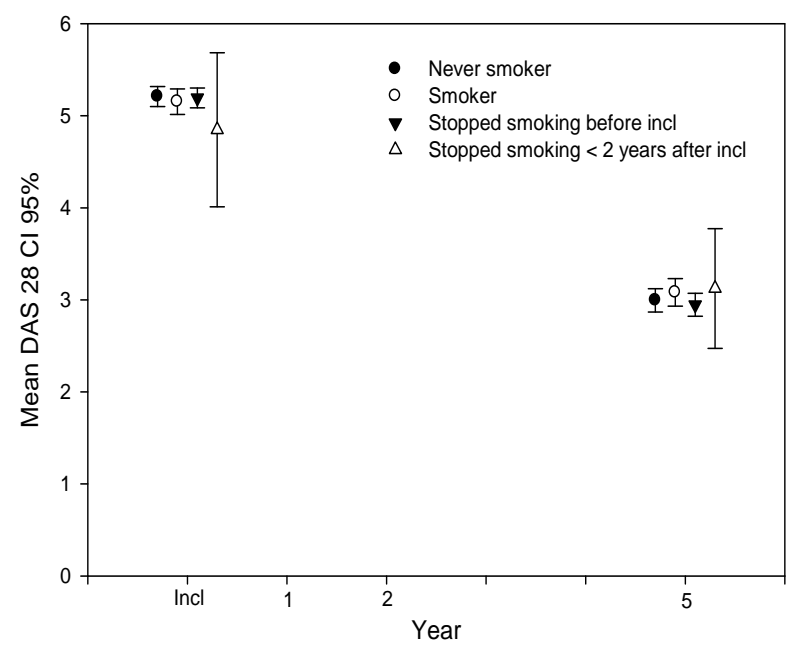

Fig. (2). Mean DAS28 (with 95\% CI) at up to 5 years of follow-up, stratified according to smoking status.

There were no statistical differences in EULAR response at 2, 5 or 8 years of follow-up between the different smoking categories (data not shown). This did not change after stratification according to gender.

\section{Multiple Logistic Regression Analysis}

The outcome of the multiple logistic regression analyses was EULAR good $v s$ no or moderate response at 8 years $(\mathrm{n}=$ 560). The patients who had stopped smoking $>7$ years from inclusion were excluded in this analysis. Cessation of smoking after inclusion in the study was independently associated with a poor prognosis (OR 0.44 , 95\% CI $0.22-$ $0.86, \mathrm{p}=0.02$ ) (Table 2). Male sex and higher DAS28 were independently associated with a better prognosis, and RF positivity and the SEI class "Other" were independently associated with poorer prognosis. The results did not change when including the patients who had stopped smoking $>7$ years from inclusion as current smokers, with the exception that SEI class "Other" was then no longer statistically significant.

\section{Stratification by Anti-CCP Antibody Status}

We had anti-CCP antibody data on 388 patients (162 were anti-CCP antibody negative and 226 were anti-CCP antibody positive). Anti-CCP antibody data were available for 51 patients who stopped smoking after inclusion in the study (26 (51\%) were anti-CCP negative and 25 (49\%) were anti-CCP positive). With stratification for anti-CCP antibody status, there were no significant differences in EULAR 
response between the different smoking categories at up to 8 years of follow-up (data not shown).

Table 2. Multiple Logistic Regression Analysis with Outcome EULAR Good $v$ s No or Moderate at 8 Years $N=560$

\begin{tabular}{|c|c|c|c|}
\hline Variable & OR & $\mathbf{9 5 \% ~ C I ~}$ & P \\
\hline \hline Current smokers & 0.62 & $0.37-1.04$ & 0.07 \\
\hline Smoking cessation before inclusion & 0.73 & $0.48-1.10$ & 0.13 \\
\hline Smoking cessation after inclusion & 0.44 & $0.22-0.86$ & 0.02 \\
\hline Age at inclusion & 0.99 & $0.97-1.00$ & 0.07 \\
\hline Disease duration (months) & 0.99 & $0.94-1.03$ & 0.53 \\
\hline DAS28 & 1.19 & $1.03-1.37$ & 0.02 \\
\hline Male sex & 2.20 & $1.44-3.36$ & 0.0001 \\
\hline SEI lower white collar & 0.97 & $0.67-1.41$ & 0.87 \\
\hline SEI upper white collar & 0.83 & $0.40-1.71$ & 0.61 \\
\hline SEI self-employed & 0.52 & $1.12-2.20$ & 0.37 \\
\hline SEI Other & 0.25 & $0.06-0.98$ & 0.047 \\
\hline RF & 0.60 & $0.41-0.87$ & 0.007 \\
\hline
\end{tabular}

The variables were baseline data for RF, age, sex, DAS28, and disease duration. Smoking and socioeconomic status were assessed from the 2010 questionnaire. The references for smoking class were never-smokers and for socioeconomic class, manual workers. The patients who stopped smoking after 7 years after enrolment are excluded in this analysis.

DAS28 = Disease Activity Score $(28$ joints $), \mathrm{RF}=$ rheumatoid factor, SEI= socioeconomic class.

\section{Stratification for Pack-Year Status}

Pack-year data were available for 507/617 (82\%) previous smokers including the patients who had stopped smoking after inclusion, and 195/231 (84\%) of the current smokers. Regarding EULAR response for heavy smokers (> 20 pack-years), at 2 years, $33 \%$ of the heavy smokers who had stopped smoking after inclusion had no EULAR response, as did $17 \%$ of current heavy smokers and $7 \%$ of heavy smokers who had stopped smoking before inclusion ( $p$ $=0.03)$. For heavy smokers, stopping smoking after inclusion was thus associated with poorer prognosis at 2 years of follow-up. For the rest of the pack-year strata $(<10$ and 10-20 pack-years), there were no statistically significant differences in EULAR response between the smoking categories.

\section{Comparison of "Early Stoppers" and "Late Stoppers"}

The patients who stopped smoking after inclusion but $\leq 2$ years from inclusion $(\mathrm{n}=30)$ were compared with patients who stopped smoking $>2$ years from inclusion $(\mathrm{n}=94)$. There were no statistically significant differences in EULAR response between these categories of smoking cessation up to 8 years (data not shown). Stratification for pack-years did not change these results. There was only a statistically significant difference in absolute DAS28 values between these two groups at 1 year: mean DAS28 for "early stoppers" was 3.0 as compared to 3.6 for "late stoppers" $(\mathrm{p}=0.04)$.
The Effect of the Time Elapsed from Cessation of Smoking Before Inclusion on Disease Activity at Inclusion

We also studied the effect of time (in years) elapsed from cessation of smoking before inclusion on disease activity at inclusion. We could not find any effect of the time point at which the patients stopped smoking before inclusion into the study on HAQ, VAS scores, DAS28 (Fig. 3), or the individual variables of DAS28 at inclusion (DAS28: $r_{\mathrm{s}}=$ $0.07, p=0.13$; HAQ: $r_{s}=0.07, p=0.12$; VAS pain: $r_{s}=0.03$, $\mathrm{p}=0.49$; VAS global: $\mathrm{r}_{\mathrm{s}}=0.06, \mathrm{p}=0.22 ; \mathrm{CRP}: \mathrm{r}_{\mathrm{s}}=0.06, \mathrm{p}=$ 0.17 ; ESR: $r_{s}=0.08, p=0.11 ;$ SJC: $r_{s}=-0.06, p=0.21$; and TJC: $\left.\mathrm{r}_{\mathrm{s}}=0.04, \mathrm{p}=0.40\right)$.

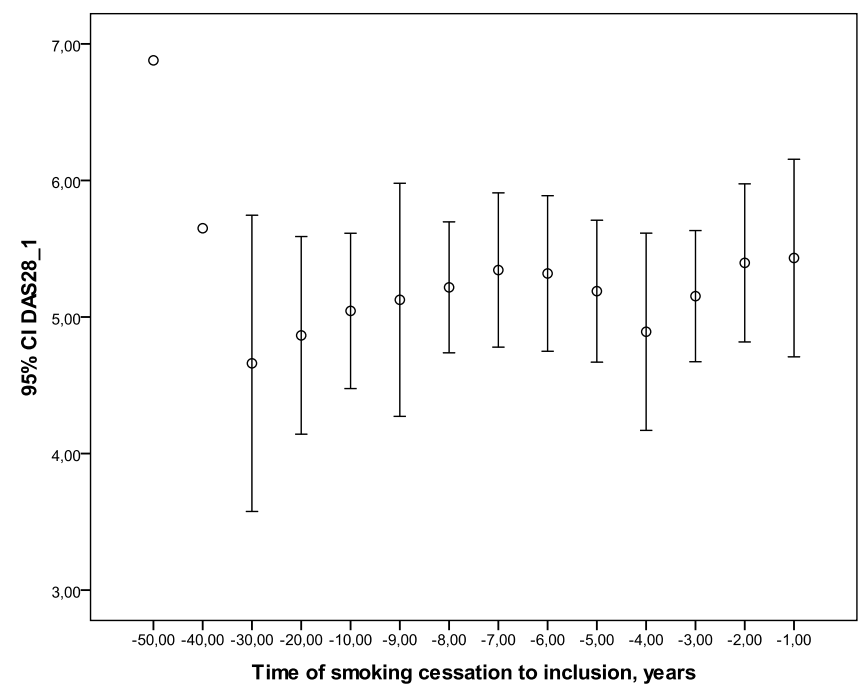

Fig. (3). Time between earlier cessation of smoking (in years) and inclusion in the study, and its correlation with mean DAS28 at inclusion $\left(\mathrm{r}_{\mathrm{s}}=0.07, \mathrm{p}=0.13\right)$.

\section{DISCUSSION}

This is to our knowledge the second study to investigate the effect of cessation of smoking on disease activity in early and established RA in a large longitudinal observational cohort. We could confirm the results from the CORRONA study in the US [13], namely that stopping smoking after inclusion in the study did not have a positive effect on EULAR outcome, HAQ or the individual variables of the DAS28. Stopping smoking after onset of RA was an independent risk factor for poor EULAR response at 8 years. We have previously reported from this same material that current smokers did poorly in a one-year follow-up [2], and others have reported similar results [3-12]. However, in this study we could see no differences in EULAR response or absolute DAS28 values, or the individual variables of the DAS28 stratified for different smoking strata, with up to 15 years of follow-up. There may be several reasons for these findings. The patients who stopped smoking may have been a selected group, for example with more comorbidities or other factors that we could not adjust for. We may also possibly be measuring factors that are related to poor health other than joint inflammation in RA patients who smoke [8]. We have previously reported that RA patients who smoke and who are starting anti-TNF therapy have a poorer 
prognosis, but the results depended on the outcome measure used and the time of follow-up, even during the first year of anti-TNF treatment [8]. The immunological process in RA starts several years before the actual clinical onset of RA [22, 23]. In RA patients who smoke, this immunological disease process may be irreversible even after they stop smoking, or that smoking enhances this patho-immunological process in some other way than by contributing to production of antiCCP antibodies. Ideally, combining shared epitope data and anti-CCP serological data could be used to identify the patients with RA that is triggered by smoking, and it might be possible to study the effects of cessation of smoking specifically in these patients. However, shared epitope did not influence the poorer response of smokers to methotrexate and biologics in another large Swedish RA study [9]. The present results clearly need to be verified using larger material, preferably with adjustment for anti-CCP serology and shared epitope.

We also compared patients who had stopped smoking early ( $\leq 2$ years after inclusion) with patients who stopped smoking later on, but we could not see any differences between these groups either in EULAR outcome or absolute DAS28 values. However, heavy smokers who stopped smoking after inclusion had poorer EULAR response at 2 years. These heavy smokers were probably a selected patient population and may have had more comorbidities and/or worse general health status.

We could not find any effect of the time of cessation of smoking (in years) before inclusion in the study on disease activity or function at inclusion. This latter finding is consistent with our earlier results using the same material [2] and those of others $[3,9]$ that previous smoking does not influence disease activity initially.

Our results obviously cannot be used to advocate smoking in RA and it should be stressed that all RA patients need to stop smoking, in particular because of the high risk of cardiovascular mortality and morbidity in RA patients $[24,25]$ and the association of smoking with vasculitis and noduli in RA [26, 27].

One of the major strengths of this study is the large and well-documented material, with a long follow-up of 15 years and relatively frequent follow-up visits during the first 2 years. We also had extensive pack-year data and data on socioeconomic class. Our patients were mainly treated with DMARDs (ie. non-biologics), although the proportion of patients on biologics increased to $23 \%$ at 15 years of followup. As treatment with DMARDs and biologics was only recorded at the follow-up visits in this study, further studies are needed to analyze the effect of stopping smoking on disease activity in RA patients being treated with biologics.

It is possible that our study lacked power, as only 127 patients stopped smoking after inclusion. There may also have been a selection bias for these patients. This study was started when smoking was not yet recognized as a poor prognostic factor in RA, and it may be so that the patients who were most actively encouraged to stop smoking may have had more comorbidities. The deceased patients may also have smoked more. However, there were no differences in baseline data and there were few differences in DMARD or glucocorticoid treatment in these patients as compared to the patients in the other smoking categories. We lacked data on shared epitope and had anti-CCP antibody status in only a minority of cases, so we could not adjust for these factors fully. One of the limitations of this study was that the data on smoking and ceasing smoking was retrospective, which may have caused misclassification of some patients due to problems with recall. There were some differences in attrition; the patients with incomplete data at all follow-up points had received less glucocorticoids than patients with complete data. On the other hand, patients with incomplete data had more often received DMARDs, combination treatment, and biologics. A total of $34 \%$ of the patients did not receive DMARD treatment at 15 years. Sixty-one per cent not treated with DMARDs at 15 years had good EULAR response, thus a majority of these patients had low disease activity. Lack of response to the 2010 questionnaire was associated with higher disease activity and smoking in our study, thus confirming other studies on attrition and nonresponse to questionnaires [28, 29]. We also attempted to analyze disease activity over time by using mixed models, but the assumption for this statistical model was not fulfilled so this method could not be used.

\section{CONCLUSION}

In summary, this is the second study to investigate the effect of stopping smoking after onset of RA on disease activity in RA. Cessation of smoking after onset of RA disease did not influence the poor prognosis of smokers. However, the patients who stopped smoking after onset of RA may have been a selected patient population, and our results must be verified in larger studies, preferably with adjustment for shared epitope and anti-CCP serology. The time in years from cessation of smoking before inclusion in the study did not influence disease activity at inclusion. Our results certainly cannot be used to advocate smoking in RA and all RA patients should be encouraged to stop smoking. Further studies on the effects of cessation of smoking on the health of RA patients are needed.

$$
\begin{array}{ll}
\text { ABBREVIATIONS } \\
\text { HAQ } & =\text { Health Assessment Questionnaire } \\
\text { VAS } & =\text { Visual analog scale } \\
\text { DMARDs } & =\text { Disease-modifying anti-rheumatic drugs } \\
\text { DAS28 } & =\text { Disease Activity Score (28 joints) } \\
\text { ESR } & =\text { Erythrocyte sedimentation rate } \\
\text { CRP } & =\text { C-reactive protein } \\
\text { RF } & =\text { Rheumatoid factor } \\
\text { SEI } & =\text { Socioeconomic class }
\end{array}
$$

\section{CONFLICTS OF INTEREST}

Dr. M.K. Söderlin has received speaking fees for educational events from Abbott $(<\$ 5,000)$ and MSD $(<$ $\$ 2,000)$ and speaking and consultation fees from Pfizer $(<$ $\$ 10,000)$. Dr. Stefan Bergman has received consultation fees from Pfizer $(<\$ 5,000)$.

\section{ACKNOWLEDGEMENTS}

We thank Jan-Åke Nilsson for invaluable help with the statistical analyses. We thank all the staff and hospitals in the BARFOT study for provision of the data. 


\section{REFERENCES}

[1] Kallberg H, Padyukov L, Plenge RM, et al. Gene-gene and geneenvironment interactions involving HLA-DRB1, PTPN22, and smoking in two subsets of rheumatoid arthritis. Am J Hum Genet 2007; 80(5): 867-75.

[2] Soderlin MK, Petersson IF, Bergman S, Svensson B. Smoking at onset of rheumatoid arthritis (RA) and its effect on disease activity and functional status: experiences from BARFOT, a long-term observational study on early RA. Scand J Rheumatol 2011; 40(4): 249-55.

[3] Saevarsdottir S, Wallin H, Seddighzadeh M, et al. Predictors of response to methotrexate in early DMARD naive rheumatoid arthritis: results from the initial open-label phase of the SWEFOT trial. Ann Rheum Dis 2011; 70(3): 469-75.

[4] Manfredsdottir VF, Vikingsdottir T, Jonsson T, et al. The effects of tobacco smoking and rheumatoid factor seropositivity on disease activity and joint damage in early rheumatoid arthritis. Rheumatology (Oxford, England) 2006; 45(6): 734-40.

[5] Papadopoulos NG, Alamanos Y, Voulgari PV, Epagelis EK, Tsifetaki N, Drosos AA. Does cigarette smoking influence disease expression, activity and severity in early rheumatoid arthritis patients? Clin Exp Rheumatol 2005; 23(6): 861-6.

[6] Wolfe F. The effect of smoking on clinical, laboratory, and radiographic status in rheumatoid arthritis. J Rheumatol 2000; 27(3): 630-7.

[7] Saag KG, Cerhan JR, Kolluri S, Ohashi K, Hunninghake GW, Schwartz DA. Cigarette smoking and rheumatoid arthritis severity. Ann Rheum Dis 1997; 56(8): 463-9.

[8] Soderlin M, Petersson I, Geborek P. The effect of smoking on response and drug survival in rheumatoid arthritis patients treated with their first anti-TNF drug. Scand J Rheumatol 2011; 41(1): 1-9.

[9] Saevarsdottir S, Wedren S, Seddighzadeh M, et al. Patients with early rheumatoid arthritis who smoke are less likely to respond to treatment with methotrexate and tumor necrosis factor inhibitors: observations from the Epidemiological Investigation of Rheumatoid Arthritis and the Swedish Rheumatology Register cohorts. Arthritis Rheum 2011; 63(1): 26-36.

[10] Hyrich KL, Watson KD, Silman AJ, Symmons DP. Predictors of response to anti-TNF-alpha therapy among patients with rheumatoid arthritis: results from the British Society for Rheumatology Biologics Register. Rheumatology (Oxford) 2006; 45(12): 1558-65.

[11] Mattey DL, Brownfield A, Dawes PT. Relationship between packyear history of smoking and response to tumor necrosis factor antagonists in patients with rheumatoid arthritis. J Rheumatol 2009; 36(6): 1180-7.

[12] Abhishek A, Butt S, Gadsby K, Zhang W, Deighton CM. AntiTNF-alpha agents are less effective for the treatment of rheumatoid arthritis in current smokers. J Clin Rheumatol 2010; 16(1): 15-8.

[13] Fisher MC, Hochberg MC, El-Taha M, Kremer JM, Peng C, Greenberg JD. Smoking, smoking cessation, and disease activity in a large cohort of patients with rheumatoid arthritis. J Rheumatol 2012; 39(5): 904-9.

[14] Westhoff G, Rau R, Zink A. Rheumatoid arthritis patients who smoke have a higher need for DMARDs and feel worse, but they do not have more joint damage than non-smokers of the same serological group. Rheumatology (Oxford) 2008; 47(6): 849-54.

[15] Forslind K, Larsson EM, Johansson A, Svensson B. Detection of joint pathology by magnetic resonance imaging in patients with early rheumatoid arthritis. Br J Rheumatol 1997; 36(6): 683-8.

[16] Forslind K, Hafstrom I, Ahlmen M, Svensson B. Sex: a major predictor of remission in early rheumatoid arthritis? Ann Rheum Dis 2007; 66(1): 46-52.

[17] Hafstrom I, Albertsson K, Boonen A, van der Heijde DM, Landewe $\mathrm{R}$, Svensson B. Remission achieved after 2 years treatment with low dose prednisolone in addition to DMARDs in early RA is associated with reduced joint destruction still present after 4 years: an open two year continuation study. Ann Rheum Dis 2008; 68(4): 508-13.

[18] Arnett FC, Edworthy SM, Bloch DA, et al. The American Rheumatism Association 1987 revised criteria for the classification of rheumatoid arthritis. Arthritis Rheum 1988; 31(3): 315-24.

[19] Ekdahl C, Eberhardt K, Andersson SI, Svensson B. Assessing disability in patients with rheumatoid arthritis. Use of a Swedish version of the Stanford Health Assessment Questionnaire. Scand J Rheumatol 1988; 17(4): 263-71.

[20] Fries JF, Spitz P, Kraines RG, Holman HR. Measurement of patient outcome in arthritis. Arthritis Rheum 1980; 23(2): 137-45.

[21] van Gestel AM, Haagsma CJ, van Riel PL. Validation of rheumatoid arthritis improvement criteria that include simplified joint counts. Arthritis Rheum 1998; 41(10): 1845-50.

[22] Rantapaa-Dahlqvist S, de Jong BA, Berglin E, et al. Antibodies against cyclic citrullinated peptide and IgA rheumatoid factor predict the development of rheumatoid arthritis. Arthritis Rheum 2003; 48(10): 2741-9.

[23] Nielen MM, van Schaardenburg D, Reesink HW, et al. Specific autoantibodies precede the symptoms of rheumatoid arthritis: a study of serial measurements in blood donors. Arthritis Rheum 2004; 50(2): 380-6.

[24] Wallberg-Jonsson S, Ohman ML, Dahlqvist SR. Cardiovascular morbidity and mortality in patients with seropositive rheumatoid arthritis in Northern Sweden. J Rheumatol 1997; 24(3): 445-51.

[25] Symmons DP, Gabriel SE. Epidemiology of CVD in rheumatic disease, with a focus on RA and SLE. Nat Rev Rheumatol 2011; 7(7): 399-408.

[26] Turesson C, Schaid DJ, Weyand CM, et al. Association of HLA-C3 and smoking with vasculitis in patients with rheumatoid arthritis. Arthritis Rheum 2006; 54(9): 2776-83.

[27] Nyhall-Wahlin BM, Jacobsson LT, Petersson IF, Turesson C. Smoking is a strong risk factor for rheumatoid nodules in early rheumatoid arthritis. Ann Rheum Dis 2006; 65(5): 601-6.

[28] Soderlin MK, Jacobsson LT, Petersson IF, Englund M, Saxne T, Geborek P. Differences in longitudinal disease and treatment characteristics of patients with rheumatoid arthritis replying and not replying to a postal questionnaire. Experience from a biologics register in southern Sweden. J Rheumatol 2009; 36(6): 1166-9.

[29] Kauppi M, Sokka T, Hannonen P. Survey nonresponse is associated with increased mortality in patients with rheumatoid arthritis and in a community population. J Rheumatol 2005; 32(5): 807-10.

(C) Andersson et al.; Licensee Bentham Open.

This is an open access article licensed under the terms of the Creative Commons Attribution Non-Commercial License (http:/creativecommons.org/licenses/by-nc/ $3.0 /$ ) which permits unrestricted, non-commercial use, distribution and reproduction in any medium, provided the work is properly cited. 